\title{
Farmacoterapia en la Enfermedad Renal
}

\author{
Dolores Andreu Periz, José Antonio Sarria Guerrero
}

Departamento de Enfermería Fundamental y Médico Quirúrgica. Facultad de Ciencias de la Salud. Universitat de Barcelona. Barcelona. España

Uno de los pilares del tratamiento de las personas con Enfermedad Renal es la administración de drogas que aporten elementos inadecuadamente metabolizados por el riñón o minimicen otras complicaciones propias de la enfermedad ${ }^{1,2}$. Muchos medicamentos de uso habitual se metabolizan o se eliminan por vía renal, pero su farmacocinética y en algunos casos la sensibilidad a éstos se ve alterada por la enfermedad; hay cambios en la absorción, distribución y metabolismo de los fármacos y también en su excreción que modifican el nivel alcanzado a dosis normal, cambiando potencialmente su eficacia y aumentando la probabilidad de acumulación y de efectos adversos, incluida la toxicidad renal ${ }^{1-3}$.

Por otra parte, existen fármacos utilizados para dolencias frecuentes que, independientemente de requerir o no de un ajuste de dosis, son nefrotóxicos y pueden afectar a la función renal, siendo significativos los antinflamatorios no esteroideos, los aminoglucósidos 0 algunos contrastes radiológicos ${ }^{2,4}$.

Para preservar la función renal, tanto normal como patológica, es necesario tener en cuenta qué medicamentos nefrotóxicos pueden ser especialmente peligrosos en personas de edad avanzada, diabéticas o con insuficiencia cardíaca, especialmente en situaciones de deshidratación o hipovolemia. Además de mantener la ingesta de líquidos y dar los fármacos estrictamente necesarios con dosis, intervalos y tiempo adecuados, si el filtrado glomerular está alterado será necesario un ajuste de dosis y controles que detecten

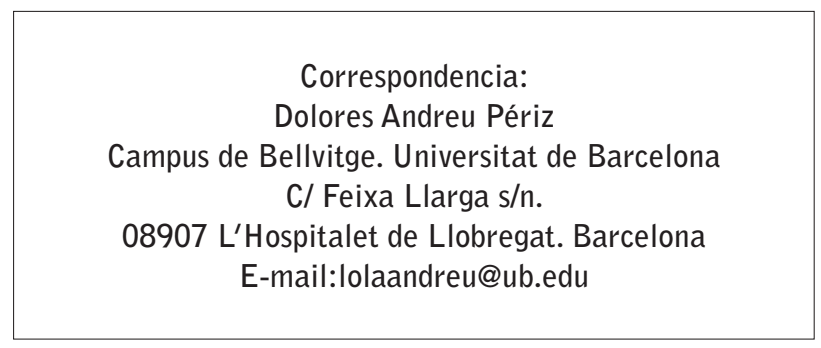

un deterioro de la función renal o la hiperpotasemia asociada a fármacos ${ }^{5}$.

La prescripción idónea y su cumplimiento puede ser especialmente complicada en los pacientes con enfermedad renal en terapia sustitutiva que plantean problemas derivados de su patología o consecuentes a los riesgos que implica la diálisis. Se ha descrito que estas personas son uno de los colectivos que toman mayor cantidad de medicación en comparación con otros enfermos crónicos. Siempre existe un cierto grado de mal cumplimiento terapéutico en todo tipo de tratamiento, pero es mucho más acusado en los tratamientos crónicos con muchas pastillas y mala tolerancia digestiva ${ }^{4,5}$. Además de fármacos para controlar enfermedades concretas como ocurre con los antidiabéticos, es casi imprescindible que los enfermos en diálisis tomen medicamentos para controlar la hipertensión, la anemia y el fósforo, suplementos vitamínicos y hierro, sin olvidar los anticoagulantes necesarios para la realización de técnicas extracorpóreas. En estos pacientes la correcta administración del fármaco se ve dificultada porque contiene moléculas dializables que se eliminan durante la terapia depuradora ${ }^{5,6}$.

El empleo adecuado de medicamentos supone un reto para los profesionales, y también para los pacientes y sus cuidadores, e implica un costo añadido muy importante en la factura sanitaria. A continuación presentamos algunos artículos cuya variada temática pone de manifiesto la complejidad del problema.

Claudio Ponticelli, Gabriele Sala, Richard J. Glassock. Drug Management in the Elderly Adult With Chronic Kidney Disease: A Review for the Primary Care Physician. Mayo Clin Proc. May 2015; 90(5):633-645. http://dx.doi.org/10.1016/j

En esta revisión se aborda el problema que plantea a los médicos de atención primaria el manejo seguro y eficaz de medicamentos frecuentemente utilizados en 
personas de edad y que pueden ser potencialmente dañinos en los pacientes con enfermedad renal. Se remarca que en la práctica clínica actual la enfermedad renal crónica (definida por una disminución mantenida a menos de $60 \mathrm{~mL} / \mathrm{min}$ por $1.73 \mathrm{~m}^{2}$ en el filtrado glomerular estimado) puede pasar desapercibida y es frecuente en personas mayores.

Se analiza en el artículo la literatura sobre gestión de medicamentos en pacientes de edad avanzada con enfermedad renal mediante la selección de los artículos publicados en Pubmed entre 1990 y 2014, utilizando las palabras clave: ancianos, enfermedad renal crónica, medicamentos, interacción medicamentosa y función renal. Se estudia en los artículos seleccionados la información sobre aspectos farmacocinéticos y farmacodinámicos, e interacciones de todos los grupos de fármacos comunes en personas mayores con comorbilidades (antihipertensivos, antibióticos, hipoglucemiantes, estatinas, etc.), también se recogen otros aspectos como la dosificación y la adherencia al tratamiento.

De esta revisión se desprende que el diseño de un régimen farmacológico adecuado en pacientes mayores con enfermedad renal es difícil porque hay poca información sobre los cambios relacionados con el envejecimiento en los perfiles farmacocinéticos y farmacodinámicos de muchas drogas, especialmente de aquellas que tienen un margen terapéutico estrecho. Agrava el problema el cumplimiento deficiente del régimen del tratamiento.

Las recomendaciones de los autores para para reducir el riesgo de eventos adversos son: 1) comenzar con dosis bajas y aumentarlas lentamente; la dosis inicial de muchos fármacos se debe fijar en la mitad de la dosis adulta normal, excepto en situaciones críticas; 2) el médico debe utilizar un número limitado de medicamentos y estar familiarizado con sus indicaciones y contraindicaciones especialmente en pacientes de edad avanzada; 3 ) el tratamiento debe ser tan simple y el número de dosis diarias debe ser tan pequeño como sea posible; 4) en las visitas de control, es necesario pedir a los pacientes una lista de los medicamentos que están tomando, la dosis y frecuencia con que los toman y cualquier evento adverso que hayan observado; 5) se ha de revisar regularmente la función renal y la creatinina en suero; y 6) una buena comunicación entre el cuidador, el paciente, y los profesionales que prescriben y controlan a estos pacientes es crucial para evitar las complicaciones derivadas del uso de fármacos.
Andrea L. Woodland, Sean W. Murphy, Bryan M. Curtis, Brendan J. Barrett. Costs Associated With Intravenous Darbepoetin Versus Epoetin Therapy in Hemodialysis Patients: A Randomized Controlled Trial. Canadian Journal of Kidney Health and Disease, 4: 1-10 Doi: 10.1177/2054358117716461

Los agentes estimulantes de la eritropoyesis han mejorado espectacularmente el manejo de la anemia, y por ende la calidad de vida de los pacientes, siendo muy numerosos los estudios que demuestran su eficacia. En este artículo se aborda un aspecto económico del tratamiento, pues los autores afirman que no están claras las diferencias entre los costos de los dos fármacos disponibles en Canadá: la epoetina alfa (EPO) y la darbepoetina alfa (DA). La EPO y la DA se consideran igualmente eficaces para lograr niveles óptimos de hemoglobina con diferentes dosificaciones, por lo que se hace difícil compararlas con criterios económicos. Con el objetivo de conocer los costes y determinar la ratio de conversión de dosis se llevó a cabo un ensayo controlado seleccionado al azar 50 pacientes adultos en hemodiálisis durante un período de 12 meses tratados con estimulantes de la eritropoyesis por vía endovenosa. El manejo de la anemia y la dosificación de estimulantes de la eritropoyesis se hicieron teniendo en cuenta las guías clínicas canadienses. Además del coste en dólares canadienses se determinaron la ratio de conversión de dosis, los índices de anemia, las dosis de hierro y su costo, y los cambios en las dosis.

Los resultados del estudio demuestran que el costo promedio fue de 4179 dólares (rango 2416-5955 dólares) para la EP0 y 2303 dólares (rango 1178-4219 dólares) para DA. La ratio de conversión de dosis fue de 360:1 (con un Intervalo de Confianza IC del 95\% de 262-457:1) a los 3 meses y a los 6 meses de 382:1 (IC del 95\%: 235-529:1). No hubo diferencias significativas entre los dos grupos respecto a la transferrina en la dosis semanal de hierro, hemoglobina y saturación y ferritina sérica. El número de cambios de dosis y el tiempo de estabilidad de la hemoglobina fueron similares.

Los autores señalan como limitaciones de su estudio que estos resultados no son extrapolables a las unidades de hemodiálisis que utilicen otras guías para el manejo de la anemia, o a los pacientes a los que se administra estimulantes de la eritropoyesis por vía subcutánea. Por otra parte, las cifras de hemoglobina óptimas utilizadas en el estudio fueron ligeramente superiores a las recomendadas hoy en día, por lo que es posible que las dosis usadas de fármacos fueron 
también mayores, por tanto, el ahorro estimado para la darbopoetina podría diferir ligeramente en la práctica. Teniendo en cuenta estas limitaciones los autores concluyen que el costo por paciente y año de la darbopoetina es de 1876 dólares canadienses (unos 1300 euros) menos que la epoyetina.

Hauber B, Caloyeras J, Posner J, Brommage D, Belozeroff V, Cooper K. Hemodialysis patients' preferences for the management of secondary hyperparathyroidism. BMC Nephrol. 2017 Jul 28;18(1):254. doi: 10.1186/s12882-017-0665-8

Este artículo define la percepción del paciente como uno de los elementos clave para un manejo óptimo de la enfermedad renal, puesto que su comprensión de los problemas y sus preferencias son necesarias para la toma compartida de las decisiones respecto a su tratamiento. El objetivo de este estudio fue conocer las preferencias de pacientes respecto el tratamiento del hiperparatiroidismo secundario asociado a la enfermedad renal.

Tras una revisión de la literatura clínica y la información obtenida de un grupo focal de pacientes se diseñó un cuestionario del que se hizo una valoración mediante una prueba pretest en un amplio grupo de pacientes antes de su administración a una muestra más amplia. La National Kidney Foundation invitó en los Estados Unidos a personas sometidas a hemodiálisis a participar en la encuesta. Los encuestados eligieron entre tres alternativas hipotéticas de tratamiento para el hiperparatiroidismo (dos médicas y una quirúrgica) en una serie de preguntas que se definieron por atributos de seguridad, tolerabilidad, su efecto sobre los análisis y sobre los síntomas, el modo de administración y los costos. Se incluyeron ítems para cuantificar las molestias relativas a la cirugía y se utilizó un modelo de regresión logística para evaluar la importancia relativa condicional de los atributos.

Contestaron al cuestionario 200 pacientes, de sus respuesta se desprende que lo más importantes para los encuestados fue si tratamiento es médico o quirúrgico y su costo. Los pacientes tenían preferencias estadísticamente significativas para los atributos de eficacia relacionados con el manejo de los síntomas y los resultados de los análisis cínicos, pero dieron menos importancia a los atributos relacionados con el modo de administración y los efectos secundarios. El atributo que evaluaron como más preocupante fue el riesgo de mortalidad quirúrgica.
A la vista de estos resultados los autores llegan a la conclusión que los pacientes que están en hemodiálisis y presentan hiperparatiroidismo entienden la enfermedad y prefieren tratamiento claros y mesurables.

Nynke G. L. Jager, Anthe S. Zandvliet, Daniel J. Touw, Erik L. Penne. Optimization of anti-infective dosing regimens during online haemodiafiltration. Clinical Kidney Journal, 2017, 10 ( 2): 282-29 doi: 10.1093/ckj/sfx009

La hemodiafiltración en línea se utiliza cada vez más en la práctica clínica como una modalidad de diálisis intermitente habitual, puesto que la suma de difusión y convección optimiza la eliminación de toxinas urémicas. La diálisis repercute en la farmacocinética de medicamentos habituales para los pacientes renales y son numerosos los estudios sobre el comportamiento de estos fármacos y su dosificación en la hemodiálisis convencional. Sin embargo, hay pocos datos sobre la necesidad de ajustes específicos para la hemodiafiltración. Esto puede ser especialmente importante en pacientes sometidos a terapia anti infecciosa, puesto que una inadecuada dosis puede ser inefectiva 0 y promover resistencias bacterianas. Con el fin de actualizar los conocimiento sobre la adecuada dosificación de algunos fármacos en pacientes sometidos a hemodiafiltración el artículo analiza los factores que determinan el aclaramiento de las drogas en el tratamiento con diálisis: a) características de la diálisis como el coeficiente de ultrafiltración, superficie y composición de la membrana, los flujos, el tiempo y la frecuencia de tratamiento; b) características del fármaco como su peso molecular, unión con proteínas, volumen de distribución y vía de eliminación y c) características del paciente como su situación clínica, valores de albúmina, función renal residual y peso. Se analizan las principales características que determinan el aclaramiento de agentes antibacterianos, antimicóticos y antivirales, se da información de cómo se puede ajustar las dosis y se comenta el valor añadido que supone monitorizar los niveles de fármacos. Este trabajo proporciona en su conjunto una guía útil para la optimización de la dosificación de agentes antiinfecciosos en pacientes sometidos a hemodiafiltración.

Recibido: 25 junio 2017

Revisado: 14 julio 2017

Modificado: 17 julio 2017

Aceptado: 21 julio 2017 


\section{Bibliografía}

1. Lorenzo Sellares V, Torres Ramírez A, Hernández Marrero D, Ayus JC (eds.). Manual de Nefrología Clínica. $2^{\mathrm{a}}$ ed. Madrid: Harcourt-Elsevier Science; 2002

2. Arias Rodríguez, M. Nefrología Clínica. Madrid. Editorial Panamericana; 2014.

3. SEN, semFYC, SEMG, Semergen, y otras sociedades científicas. Documento de consenso sobre la Enfermedad Renal Crónica. 27 de noviembre 2012. [Consultado 30 Abril 2017]. Consultado en: http://www.senefro.org/ modules/news/images/v._5.doc_consenso_final__131212_ copyl. pdf
4. Bonal J, Bonafont X. Ajuste de la dosis de medicamentos en los enfermos con insuficiencia renal. Butlletí d'informació terapéutica. 2007; 19 (4):19-24.

5. Álvarez de Lara, MA; García Montemayor, Victoria. Ajuste de Fármacos en la Insuficiencia Renal. En: Lorenzo V, López Gómez JM (Eds) Nefrología al Día. [Consultado 5 Mayo 2017). Disponible en: http://www.revistanefrologia.com/ es-monografias-nefrologia-dia-articulo-ajustefarmacos-insuficiencia-renal-159

6. Daugirdas, JT, Ing TS. Manual de diálisis. $5^{a}$ Eds. Barcelona Lippincott Williams \& Wilkins (Espaก̃a); 2015. 